\title{
Prevenção primária de delirium em idosos sob terapia intensiva: uma revisão
}

\section{integrativa}

\author{
Primary prevention of delirium in elderly patients under intensive care: an integrative review \\ Prevención primaria del delirio en pacientes ancianos en cuidados intensivos: una revisión \\ integradora
}

Recebido: 26/08/2021 | Revisado: 01/09/2021 | Aceito: 09/09/2021 | Publicado: 12/09/2021

\author{
Thyago de Oliveira Afonso \\ ORCID: https://orcid.org/0000-0001-7616-9011 \\ Universidade Federal de Pernambuco, Brasil \\ E-mail: thyago.oafonso@gmail.com \\ Samuel Lopes dos Santos \\ ORCID: https://orcid.org/0000-0003-3375-9171 \\ Universidade Federal do Piauí, Brasil \\ E-mail: samuellopes121314@gmail.com \\ Gustavo Baroni Araújo \\ ORCID: https://orcid.org/0000-0002-3162-7477 \\ Universidade Estadual de Londrina, Brasil \\ E-mail: gustavobaroni13@hotmail.com \\ Amanda Costa Maciel \\ ORCID: https://orcid.org/0000-0002-2668-037X \\ Universidade Federal de Sergipe, Brasil \\ E-mail: amandacmaciel@hotmail.com \\ Kellyane Folha Gois Moreira \\ ORCID: https://orcid.org/0000-0001-6451-5925 \\ Universidade Federal do Piauí, Brasil \\ E-mail: kelly_folha@hotmail.com \\ Andressa Leite Rodrigues Batista \\ ORCID: https://orcid.org/0000-0003-3109-6140 \\ Centro Universitário Santo Agostinho, Brasil \\ E-mail: abdressaleite@gmail.com \\ Ricardo Pessoa Rocha Melo \\ ORCID: https://orcid.org/0000-0002-7868-9325 \\ Universidade Federal do Piauí, Brasil \\ Email: ricardo.fisio5@hotmail.com \\ Cedric Adam Spíndola de Araújo Viana \\ ORCID: https://orcid.org/0000-0003-0404-1144 \\ Centro Universitário UniFacid, Brasil \\ E-mail: cedricadam8@hotmail.com \\ Joelma Maria dos Santos da Silva Apolinário \\ ORCID: https://orcid.org/0000-0001-9521-9432 \\ Centro Universitário Maurício de Nassau, Brasil \\ E-mail: jo.silva00@hotmail.com \\ Lara Maria Teles Guimarães Falcão \\ ORCID: https://orcid.org/0000-0003-0765-6929 \\ Centro Universitário Uninovafapi, Brasil \\ E-mail: lara.m.t.g.f@hotmail.com \\ Caroliny Ferreira Lira \\ ORCID: https://orcid.org/0000-0001-7907-9791 \\ Secretaria de Saúde do Recife, Brasil \\ E-mail: liracarolinyferreira@gmail.com \\ Layanne Cavalcante de Moura \\ ORCID: https://orcid.org/0000-0003-2781-1076 \\ Faculdade Integral Diferencial, Brasil \\ E-mail: layannecavalcante @ hotmail.com \\ Filipe Caleb Maia \\ ORCID: https://orcid.org/0000-0002-2148-0707 \\ Secretaria Municipal de Saúde de Pará de Minas, Brasil \\ E-mail: filipecaleb@gmail.com \\ Adrielle Pieve de Castro \\ ORCID: https://orcid.org/0000-0002-1260-5619 \\ Universidade Federal de Minas Gerais, Brasil \\ E-mail: adriellepieve@ hotmail.com
}


Paulo Henrique Barcelos

ORCID: https://orcid.org/0000-0002-1289-4553

Universidade Federal de São João Del-Rei, Brasil

E-mail: paulomedufsj@gmail.com

Karina Aparecida Resende

ORCID: https://orcid.org/0000-0001-7812-7042 Centro Universitário de Lavras, Brasil E-mail: karinaresendeufsj@gmail.com

Angelo Elias Meri Júnior

ORCID: https://orcid.org/0000-0002-8847-3915 Universidade Federal de São João Del-Rei, Brasil E-mail: angeloemj@gmail.com

Adrila David Meri

ORCID: https://orcid.org/0000-0002-1158-4246

Hospital e Maternidade Santa Mônica, Brasil

E-mail: adrila_adm@hotmail.com

Ana Cristina La Guárdia Custódio Pereira

ORCID: https://orcid.org/0000-0002-6674-9052 Universidade Federal de São João Del-Rei, Brasil E-mail: anaclaguardia@gmail.com

\title{
Resumo
}

Introdução: O delirium é um estado flutuante da cognição e da consciência, que pode cursar com perda de memória, irritabilidade, colaboração prejudicada e ciclo sono-vigília desregulado. A prevalência de delirium em Unidades de Terapia Intensiva (UTIs) está relacionada a longo período de tempo sob ventilação mecânica, presença de dor importante, hipotensão e maior mortalidade. A população idosa hospitalizada é atingida em cerca de $15 \%$ por essa morbidade, variando para aproximadamente $42 \%$, a depender de comorbidades associadas. Diante disso, é imprescindível que se reúna evidências acerca da prevenção do delirium em idosos internados em UTI. Metodologia: Trata-se de uma revisão integrativa de literatura feita nas bases de dados SciELO, Medline e LILACS, com descritores MESH e visando a selecionar artigos publicados de 2017 a 2021, em qualquer idioma, que estivessem no fulcro do objetivo proposto. Os critérios de exclusão foram: ser artigo de opinião, de revisão, metanálise, protocolo de trial, pré-print ou relato de caso. Também excluiram-se aqueles artigos cujo resumo, título ou metodologia não condiziam com o proposto nesta revisão. Resultados e Discussão: De 71 artigos selecionados inicialmente, resgatou 14 após aplicação dos critérios de exclusão. 7 foram randomizações e 7 estudos observacionais. Fármacos como a melatonina, sinvastatina, xenônio, ramelteona, dexmedetomidina e outros foram avaliados em populações idosas internas em UTI com diferentes comorbidades como potenciais medicações profiláticas ao delirium. Além disso, medidas não farmacológicas, como a redução de ruídos noturnos, também foram descritas. Destacamos as limitações impostas pela própria metodologia da revisão integrativa tem suas implicações sobre os resultados e conclusões deste estudo. Conclusão: Há formas farmacológicas e não-farmacológicas que diminuem a incidência do delirium em pacientes idosos internados em UTI, e que a heterogeneidade dos estudos que constroem essas evidências deve ser considerada, de forma a favorecer a construção de metanálises que as avalie estatisticamente.

Palavras-chave: Delirium; Terapia Intensiva; Idoso.

\begin{abstract}
Introduction: Delirium is a fluctuating state of cognition and consciousness, which can lead to memory loss, irritability, impaired collaboration and a dysregulated sleep-wake cycle. The prevalence of delirium in Intensive Care Units (ICUs) is related to a long period of time under mechanics, presence of severe pain, hypotension and higher mortality. A hospitalized elderly population is affected in about $15 \%$ by this morbidity, ranging to approximately $42 \%$, dependent on associated comorbidities. Therefore, it is essential to gather evidence on the prevention of delirium in elderly patients admitted to the ICU. Methodology: This is an integrative literature review carried out in the SciELO, Medline and LILACS databases, with MESH descriptors and selected from articles published from 2017 to 2021, in any language, that were at the heart of the proposed objective. Exclusion criteria were: being an opinion article, review article, meta-analysis, trial protocol, pre-print or case report. Articles whose abstract, title or methodology did not match what was proposed in this review were also excluded. Results and discussion: Of 71 articles bulletins bulletins, 14 retrieved after applying the exclusion criteria. 7 were randomizations and 7 observational studies. Drugs such as melatonin, simvastatin, xenon, rameltheone, dexmedetomidine and others were added in elderly women in ICU with different comorbidities as potential prophylactic medications for delirium. In addition, non-pharmacological measures, such as night noise reduction, were also eliminated. We highlight the limitations imposed by the integrative review methodology itself, which has its result on the results and research of this study. Conclusion: There are pharmacological and non-pharmacological forms that reduce the impact of delirium in elderly patients admitted to the ICU, and that the heterogeneity of studies that build evidence must be considered, in order to favor the construction of a meta-analysis that statistically assesses them.
\end{abstract}

Keywords: Delirium; Intensive therapy; Seniors. 


\begin{abstract}
Resumen
Introducción: El delirio es un estado fluctuante de cognición y conciencia que puede provocar pérdida de memoria, irritabilidad, alteración de la colaboración y un ciclo de sueño-vigilia desregulado. La prevalencia de delirio en Unidades de Cuidados Intensivos (UCI) está relacionada con un largo período de tiempo en mecánica, presencia de dolor severo, hipotensión y mayor mortalidad. Una población anciana hospitalizada se ve afectada en aproximadamente un $15 \%$ por esta morbilidad, que varía hasta aproximadamente un $42 \%$, dependiendo de las comorbilidades asociadas. Por tanto, es fundamental recopilar evidencias sobre la prevención del delirio en pacientes ancianos ingresados en UCI. Metodología: Se trata de una revisión integradora de la literatura realizada en las bases de datos SciELO, Medline y LILACS, con descriptores MESH y seleccionada a partir de artículos publicados de 2017 a 2021, en cualquier idioma, que estuvieron en el centro del objetivo propuesto. Los criterios de exclusión fueron: ser un artículo de opinión, artículo de revisión, metanálisis, protocolo de ensayo, preimpresión o informe de caso. También se excluyeron los artículos cuyo resumen, título o metodología no coincidiera con lo propuesto en esta revisión. Resultados y discusión: De 71 artículos boletines boletines, 14 recuperados luego de aplicar los criterios de exclusión. 7 fueron aleatorizaciones y 7 estudios observacionales. Se agregaron fármacos como melatonina, simvastatina, xenón, rameltheona, dexmedetomidina y otros en mujeres ancianas en UCI con diferentes comorbilidades como posibles medicamentos profilácticos para el delirio. Además, también se eliminaron las medidas no farmacológicas, como la reducción del ruido nocturno. Destacamos las limitaciones que impone la propia metodología de revisión integradora, que tiene su resultado en los resultados y la investigación de este estudio. Conclusión: Existen formas farmacológicas y no farmacológicas que reducen el impacto del delirio en pacientes ancianos ingresados en UCI, y que se debe considerar la heterogeneidad de estudios que construyen evidencia, para favorecer la construcción de un metaanálisis que estadísticamente los evalúa.
\end{abstract}

Palabras clave: Delirio; Terapia intensiva; Mayores.

\title{
1. Introdução
}

O delirium é um fenômeno de causa multifatorial e definido como um estado alterado e flutuante da cognição e da consciência, causando sintomatologia neurocomportamental que cursa com colaboração prejudicada, falta de capacidade de interpretação, perda de memória, alterações emocionais importantes e ciclo sono-vigília desregulado. Nessa perspectiva, quanto menos houver estímulos à orientação, mais crítico serão os achados clínicos do paciente. O delirium é prevalente em cerca de $15 \%$ na população idosa hospitalizada, sendo um importante fator de comorbidade clínica; contudo, sua prevalência varia de acordo com o fator que motivou a internação, a exemplo disso temos cirurgias de grande porte e as cardíacas, que chegam a ter até $42 \%$ de incidência de delirium pós-operatório. Delirium pode ser hiperativo, hipoativo e misto, que é uma flutuação entre os dois primeiros tipos. O hiperativo é mais reconhecível clinicamente, pois o paciente frequentemente encontra-se irritado e em agitação psicomotora. Já o hipoativo é definido como pensamentos e reações mais lentas. (Ford et al., 2020; Faustino et al., 2016; Herling et al., 2018).

O desenvolvimento de delirium em Unidades de Terapia Intensiva (UTIs) está relacionado a longo período de tempo em ventilação mecânica, maior período de permanência na UTI e no serviço em si e maior risco de desfecho letal. Também, há fatores como idade avançada, etilismo crônico, doenças respiratórias crônicas, fortes dores, uso de contenções físicas por muito tempo e deficiência auditiva e visual. Há também fatores precipitantes de delirium, que, na UTI, destacam-se como sendo estes: iatrogenias, sepse grave, uso de benzodiazepínicos, anticolinérgicos, esteróides, privação do sono, desidratação, distúrbios metabólicos e hipotensão, por exemplo (Herling et al., 2018).

A prevenção primária do delirium pode ter caráter farmacológico e não-farmacológico, com diferentes níveis de evidência e recortes populacionais estudados, o que evidencia a necessidade de estudos que reúnam tais pesquisas e as compare entre si. Diante disso, esta revisão tem como objetivo reunir estudos clínicos que versem sobre as estratégias de prevenção primária do delirium na população idosa internada em UTIs.

\section{Metodologia}

Esta é uma Revisão Integrativa de Literatura elaborada com base na retirada de artigos das bases de dados Scientific Electronic Library Online (SciELO), Literatura Latino Americana e do Caribe em Ciências da Saúde (LILACS) e Medical 
Literature Analysis and Retrieval Sistem (Medline) em agosto de 2021. Para a realização da pesquisa na SciELO, foram utilizados os seguintes descritores MeSH e operador booleano: “Intensive Care Unit AND delirium”. Para a LILACS, aplicouse os seguintes unitermos: "Intensive Care Unit AND delirium AND prevention”. Já na Medline, os descritores foram estes: “Intensive Care Unit AND delirium AND elderly AND primary prevention”. Neste artigo, incluiu-se manuscritos científicos publicados entre 2017 e 2021 e em qualquer idioma. De início, excluiu-se artigos de opinião, de revisão, metanálises, protocolos de trials, pré-prints e relatos de caso. Não houve duplicações. Após as exclusões iniciais, foi feita leitura dos títulos e resumos e foram retirados da amostra os artigos cujo escopo não era compatível com o eixo temático deste artigo. Depois, sistematizou-se os dados dos 24 artigos resgatados nesta última etapa em uma adaptação de um instrumento de coleta de dados nacionalmente validado por Ursi (2005) (ANEXO 01), para avaliação de seus textos por completo. Ao final do processo de resgate, 14 artigos compõem os resultados desta revisão. Abaixo na figura 1, um fluxograma que sistematiza a aplicação dos critérios de exclusão (Ursi, 2005).

Figura 1 - Fluxograma que evidencia o processo de amostragem.

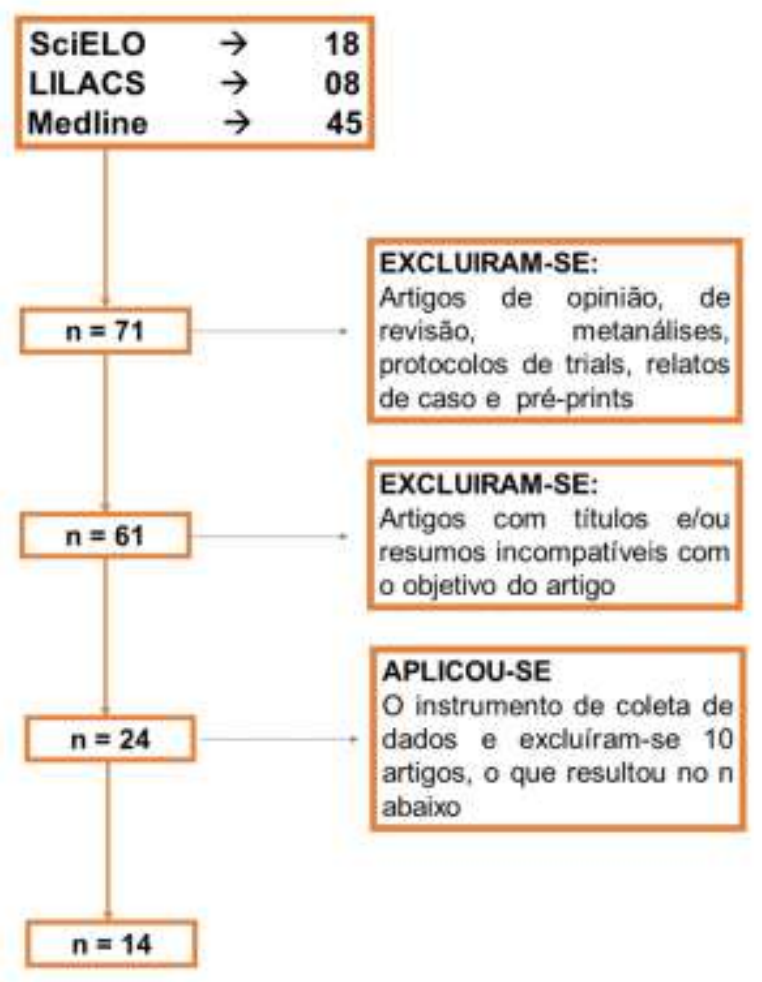

Fonte: Autores.

\section{Resultados e Discussão}

Todos os artigos selecionados são da base de dados Medline. Quanto ao ano de publicação, 28,3\% deles foi publicado em 2018, 36\% em 2017, 7\% em 2019, 21,7\% em 2020 e 7\% em 2021. Destaca-se que esta revisão, em si, é insuficiente para espelhar todo o fulcro de artigos acerca do tema proposto, pois é de naturalidade da sua metodologia que haja, a partir da pesquisa por determinados descritores, a exclusão de manuscritos científicos que poderiam ser selecionados após a aplicação dos critérios de exclusão aqui propostos. Para melhor compreensão dos resultados, os dados foram apresentados no Quadro 1, a seguir. 
Quadro 1 - Quadro que organiza as informações referentes a cada um dos artigos resgatados nessa revisão que foram selecionados após a aplicação dos critérios de exclusão.

\begin{tabular}{|c|c|c|c|}
\hline $\begin{array}{l}\text { AUTOR, ANO, } \\
\text { DESENHO }\end{array}$ & OBJETIVO & AMOSTRA & RESULTADOS E CONCLUSÕES \\
\hline $\begin{array}{l}\text { PAGE et al., 2017, } \\
\text { Ensaio } \\
\text { randomizado } \\
\text { duplo-cego } \\
\text { controlado }\end{array}$ & $\begin{array}{l}\text { Verificar se o uso de } \\
\text { sinvastatina oral previne } \\
\text { o aparecimento ou } \\
\text { aumenta o tempo livre de } \\
\text { delirium e coma em } \\
\text { pacientes sob ventilação } \\
\text { mecânica com alto risco } \\
\text { de desenvolvimento de } \\
\text { delirium. }\end{array}$ & $\begin{array}{l}142 \text { pessoas maiores de } 18 \text { anos, com } \\
\text { média de idade de } 62 \text { anos, entre } \\
\text { homens e mulheres, distribuídos } \\
\text { igualmente entre o grupo intervenção } \\
\text { e o grupo placebo. }\end{array}$ & $\begin{array}{l}\text { No dia } 14 \text { de internação em UTI, os dois } \\
\text { grupos não apresentaram significativa } \\
\text { diferença de dias em delirium ou coma. A } \\
\text { incidência de delirium no grupo sinvastatina } \\
\text { foi de } 93 \% \text { e no placebo foi de } 94 \% \text {. Para o } \\
\text { grupo que recebeu a sinvastatina, foram } 5.7 \\
\text { dias em delirium em média, e } 6.1 \text { dias para o } \\
\text { grupo placebo. Dessa forma, o estudo não } \\
\text { apoia o uso de sinvastatina para tratamento do } \\
\text { delirium. }\end{array}$ \\
\hline $\begin{array}{l}\text { COBURN et al., } \\
\text { 2018, Fase II de } \\
\text { um Ensaio Clínico } \\
\text { Randomizado } \\
\text { Duplo-cego, } \\
\text { controlado e } \\
\text { multicêntrico }\end{array}$ & $\begin{array}{l}\text { Verificar se a anestesia } \\
\text { por xenônio em cirurgias } \\
\text { de fratura de quadril em } \\
\text { idosos proporciona } \\
\text { menor incidência de } \\
\text { delírium pós-operatório } \\
\text { em comparação com a } \\
\text { anestesia feita à base de } \\
\text { sevoflurano. }\end{array}$ & $\begin{array}{l}\text { Foram selecionados } 230 \text { pacientes de } \\
\text { ambos os sexos - com prevalência } \\
\text { para o feminino ( } 75 \% \text { ), com } 75 \text { anos } \\
\text { de idade ou mais (média de } 84 \text { anos) } \\
\text { com fratura de quadril e cirurgia } \\
\text { ortopédica marcada para até } 48 \text { horas, } \\
\text { que não possuissem esquizofrenia, } \\
\text { demência, história de trauma ou } \\
\text { acidente vascular cerebral, delírio ou } \\
\text { pontuação menor que } 24 \text { no } \\
\text { Miniexame do Estado Mental. }\end{array}$ & $\begin{array}{l}\text { O estudo concluiu que a incidência de } \\
\text { delírium pós-operatório não foi } \\
\text { significativamente diferente entre o grupo que } \\
\text { recebeu xenônio e o grupo do sevoflurano. O } \\
\text { estudo aponta a necessidade de pesquisas } \\
\text { mais aprofundadas para esclarecimentos das } \\
\text { constatações elencadas a partir dos seus } \\
\text { resultados. }\end{array}$ \\
\hline $\begin{array}{l}\text { O'CONNELL et } \\
\text { al., 2018, Estudo } \\
\text { der Coorte } \\
\text { Retrospectivo }\end{array}$ & $\begin{array}{lr}\text { Comparar o risco de } \\
\text { delirium entre idosos } \\
\text { com fraturas de } \operatorname{arcos} \\
\text { costais tratados } & \text { com } \\
\text { opioides e os } & \text { com } \\
\text { analgesia regional } & \end{array}$ & $\begin{array}{l}\text { Selecionou-se } 144 \text { pacientes de } \\
\text { ambos os sexos, com média de } 74.5 \\
\text { anos de idade, admitidos na UTIs, } \\
\text { divididos em um grupo tratado com } \\
\text { opioide e outro com analgesia } \\
\text { regional. Foram excluídos os } \\
\text { pacientes com demência ou com } \\
\text { algum tipo de injúria cerebral } \\
\text { traumática. }\end{array}$ & $\begin{array}{l}\text { A prevalência de delirium na população } \\
\text { estudada foi de } 32 \% \text {, abaixo do que se } \\
\text { encontra em estudos como esse. Concluiu-se } \\
\text { que, neste estudo, a analgesia regional esteve } \\
\text { relacionada com a diminuição da incidência } \\
\text { de delirium em pacientes idosos com trauma } \\
\text { grave de tronco, bem como com a diminuição } \\
\text { da utilização de opioides sistêmicos, } \\
\text { benzodiazepínicos e antipsicóticos atípicos. } \\
\text { Os autores sinalizam a necessidade de } \\
\text { realização de estudos multicêntricos com } \\
\text { outros desenhos para que se avalie mais } \\
\text { afundo tais conclusões. }\end{array}$ \\
\hline $\begin{array}{l}\text { VARGA- } \\
\text { MARTINÉZ et al., } \\
2021, \quad \text { Estudo } \\
\text { observacional } \\
\text { multicêntrico } \\
\text { prospectivo }\end{array}$ & $\begin{array}{l}\text { Desenvolver e validar um } \\
\text { instrumento de } \\
\text { quantificação de risco de } \\
\text { delirium } \\
\text { (DELIPRECAS) pré- } \\
\text { operatório em pacientes } \\
\text { encaminhados à cirurgia } \\
\text { cardíaca. }\end{array}$ & $\begin{array}{l}\text { Selecionou-se } 689 \text { pacientes maiores } \\
\text { de } 18 \text { anos, sendo cerca de } 67 \% \text { com } \\
\text { mais de } 65 \text { anos, com média de idade } \\
\text { da população geral de } 70.7 \text { anos, com } \\
\text { recente cirurgia cardíaca e internados } \\
\text { em UTI. A amostra foi dividida } \\
\text { igualmente, de forma randomizada, } \\
\text { em dois grupos: o que seria } \\
\text { investigado para desenho do } \\
\text { instrumento }(n=345) \text { e o outro que o } \\
\text { validaria }(n=344) \text {. }\end{array}$ & $\begin{array}{l}\text { Do primeiro grupo, } 17,1 \% \text { da amostra } \\
\text { desenvolveu delírio, enquanto houve } \\
\text { incidência em } 16,9 \% \text { da outra. O delírio mais } \\
\text { prevalente }(58,2 \%) \text { foi o hiperativo. O tipo de } \\
\text { cirurgia cardíaca não teve influência sobre a } \\
\text { incidência de delirium. Os pacientes que } \\
\text { desenvolveram delirium ficaram por mais } \\
\text { tempo em admissão e intubação. O estudo } \\
\text { ressalta que idade maior que } 65 \text { anos é fator } \\
\text { de risco para pacientes submetidos a cirurgia } \\
\text { cardíaca apresentarem delirium pós- } \\
\text { operatório e ressalta que a prevenção primária } \\
\text { é a melhor forma de tratamento contra essa } \\
\text { comorbidade. Ainda, concluíram que o } \\
\text { DELIPRECAS tem capacidade de predizer, } \\
\text { no momento pré-operatório, qual o risco de o } \\
\text { paciente de cirurgia cardíaca desenvolver } \\
\text { delirium pós-operatório. }\end{array}$ \\
\hline
\end{tabular}




\begin{tabular}{|c|c|c|c|}
\hline $\begin{array}{l}\text { VAN DE POL; } \\
\text { ITERSON; } \\
\text { MAASKANT, } \\
\text { 2017, Análise de } \\
\text { série temporal } \\
\text { interrompida }\end{array}$ & $\begin{array}{l}\text { Verificar o efeito de um } \\
\text { protocolo de redução de } \\
\text { som durante a noite na } \\
\text { incidência de delirium e } \\
\text { na qualidade do sono de } \\
\text { pacientes clinicamente } \\
\text { graves admitidos em uma } \\
\text { UTI }\end{array}$ & $\begin{array}{l}411 \text { pessoas, entre homens e } \\
\text { mulheres, com idade média de } 68,5 \\
\text { anos, com gravidade de quadro } \\
\text { clínico semelhante, admitidos em } \\
\text { UTI. }\end{array}$ & $\begin{array}{l}\text { Imediatamente, não houve mudanças na } \\
\text { incidência do delirium após o início da } \\
\text { intervenção. Mas, ao final do período do } \\
\text { estudo, no grupo pré-intervenção, o declínio } \\
\text { de delirium foi estável em } 0,9 \% \text { por período } \\
\text { de tempo. Já no grupo pós-intervenção, o } \\
\text { delirium decresceu mais - cerca de } 2,9 \% \text { - a } \\
\text { cada intervalo de tempo. Isso demonstrou que } \\
\text { a estratégia de diminuição de sons à noite é } \\
\text { efetiva em diminuir a incidência de delirium } \\
\text { nos idosos admitidos na UTI segundo a } \\
\text { metodologia deste estudo. }\end{array}$ \\
\hline $\begin{array}{l}\text { DEINER et al., } \\
2017, \quad \text { Ensaio } \\
\text { Clínico } \\
\text { Randomizado } \\
\text { Duplo-cego, } \\
\text { controlado } \\
\text { multicêntrico }\end{array}$ & $\begin{array}{l}\text { Verificar se a infusão de } \\
\text { dexmedetomidina } \\
\text { diminui a incidência de } \\
\text { delirium pós-operatório }\end{array}$ & $\begin{array}{l}189 \text { pacientes foram locados para o } \\
\text { grupo intervenção e } 201 \text { para o grupo } \\
\text { placebo. A média de idade foi de } 74 \\
\text { anos e todos tiveram cirurgias eletivas } \\
\text { não-cardíacas de grande porte com } \\
\text { anestesia geral }\end{array}$ & $\begin{array}{l}\text { Segundo os resultados desse estudo, sua } \\
\text { metodologia e as características de sua } \\
\text { amostra, o uso intraoperatório de } \\
\text { dexmedetomidina em pacientes idosos que } \\
\text { foram submetidos a cirurgias não-cardíacas } \\
\text { de grande porte não diminui a incidência de } \\
\text { delirium. A incidência desse quadro nos } \\
\text { grupos randomizados foi de } 12,2 \% \\
\text { (intervenção) e } 11,4 \% \text { (placebo). }\end{array}$ \\
\hline $\begin{array}{l}\text { HATTA et al., } \\
2017, \quad \text { Ensaio } \\
\text { Clínico } \\
\text { Randomizado } \\
\text { Duplo-cego, } \\
\text { controlado e } \\
\text { multicêntrico }\end{array}$ & $\begin{array}{l}\text { Verificar se o } \\
\text { sulvorexant é eficaz na } \\
\text { prevenção do delirium. }\end{array}$ & $\begin{array}{l}72 \text { pacientes de } 65 \text { a } 89 \text { anos, de } \\
\text { ambos os sexos, que foram admitidos } \\
\text { em UTI e que tinham expectativa de } \\
\text { vida maior que } 48 \text { horas. }\end{array}$ & $\begin{array}{l}\text { Quando administrado todas as noites, o } \\
\text { sulvorexant teve efeito protetivo contra o } \\
\text { delirium, diminuindo sua incidência na } \\
\text { população estudada. }\end{array}$ \\
\hline $\begin{array}{l}\text { BOOGARD et al., } \\
2018, \quad \text { Ensaio } \\
\text { Clínico } \\
\text { Randomizado } \\
\text { Duplo-cego } \\
\text { Placebo- } \\
\text { controlado }\end{array}$ & $\begin{array}{l}\text { Verificar se o haloperidol } \\
\text { melhora a sobrevida de } \\
\text { pacientes graves em UTI } \\
\text { com alto risco de } \\
\text { delirium. }\end{array}$ & $\begin{array}{l}1.789 \text { pacientes de ambos os sexos, } \\
\text { com pelo menos } 18 \text { anos de idade, } \\
\text { com média de idade de } 66,6 \text { anos, } \\
\text { com previsão de internação em UTI } \\
\text { por pelo menos } 2 \text { dias. }\end{array}$ & $\begin{array}{l}\text { Nesse estudo, não houve evidência de que o } \\
\text { haloperidol melhora a sobrevida dos } \\
\text { pacientes idosos internados em UTI, pois seus } \\
\text { resultados foram muito semelhantes ao } \\
\text { placebo, em } 28 \text { dias. }\end{array}$ \\
\hline $\begin{array}{l}\text { ROSA et al., } 2017, \\
\text { Estudo prospectivo } \\
\text { "antes e depois" }\end{array}$ & $\begin{array}{l}\text { Avaliar o impacto da } \\
\text { ampliação das horas de } \\
\text { visitação na incidência de } \\
\text { delirium em pacientes } \\
\text { internados em UTI. }\end{array}$ & $\begin{array}{l}286 \text { pacientes, entre homens e } \\
\text { mulheres maiores de } 18 \text { anos de } \\
\text { idade, com média de } 61.45 \text { anos de } \\
\text { idade. Foram excluídos pacientes com } \\
\text { afasia, delirium pré-existente e os que } \\
\text { estavam sob cuidados paliativos. Os } \\
\text { participantes foram divididos em dois } \\
\text { grupos, um com } 4,5 \mathrm{~h} \text { de visitação por } \\
\text { dia, e outro com } 12 \mathrm{~h} \text { de visitação por } \\
\text { dia, com visitas mais duradouras. }\end{array}$ & $\begin{array}{l}\text { O grupo que recebeu visitações por mais } \\
\text { tempo teve incidência de delirium em torno } \\
\text { de } 10 \% \text {, enquanto o grupo que recebeu visitas } \\
\text { por menor período teve cerca de } 20,5 \% \text { da } \\
\text { sua população com delirium. }\end{array}$ \\
\hline $\begin{array}{l}\text { CUCCI et al., } \\
2020 \text {, Estudo de } \\
\text { coorte } \\
\text { observacional } \\
\text { retrospectivo }\end{array}$ & $\begin{array}{l}\text { Observar o impacto do } \\
\text { período de reiniciação do } \\
\text { tratamento } \\
\text { neuropsiquiátrico após a } \\
\text { admissão hospitalar na } \\
\text { incidência do delirium } \\
\text { em pessoas internadas } \\
\text { em UTI. }\end{array}$ & $\begin{array}{l}300 \text { pessoas, de ambos os sexos, } \\
\text { admitidas em UTI e com média de } \\
\text { idade de } 65.3 \text { anos. }\end{array}$ & $\begin{array}{l}\text { Os grupos que recomeçaram o tratamento } \\
\text { neuropsicológico antes e depois de } 72 \mathrm{~h} \text { não } \\
\text { demonstraram diferenças significantes de } \\
\text { incidência de delirium. }\end{array}$ \\
\hline $\begin{array}{l}\text { NISHIKIMI et al., } \\
2018, \quad \text { Estudo } \\
\text { randomizado, } \\
\text { placebo- }\end{array}$ & $\begin{array}{l}\text { Avaliar se o Ramelteon } \\
\text { pode prevenir o delirium } \\
\text { e encurtar o período de }\end{array}$ & $\begin{array}{l}88 \text { indivíduos de ambos os sexos, } \\
\text { com } 20 \text { anos ou mais e média de } \\
\text { idade de } 68 \text { anos, internados em UTI } \\
\text { que puderam receber o ramelteon de }\end{array}$ & $\begin{array}{l}\text { O grupo que recebeu o ramelteon }(45 \\
\text { pessoas) teve } 24,4 \% \text { de incidência de } \\
\text { delirium, enquanto o grupo placebo }(43 \\
\text { pessoas) teve } 46,6 \% \text {. Logo, percebe-se que o }\end{array}$ \\
\hline
\end{tabular}




\begin{tabular}{|c|c|c|c|}
\hline $\begin{array}{l}\text { controlado, triplo- } \\
\text { cego }\end{array}$ & internação em UTI. & $\begin{array}{l}\text { forma oral ou via tubo de alimentação } \\
\text { nas primeiras } 48 \mathrm{~h} \text { de admissão na } \\
\text { UTI. }\end{array}$ & $\begin{array}{l}\text { ramelteon teve significativa relevância } \\
\text { estatística em diminuir a incidência de } \\
\text { delirium na população estudada, bem como } \\
\text { de diminuir o tempo de internação em UTI. }\end{array}$ \\
\hline $\begin{array}{l}\text { LARSEN et al., } \\
2020, \quad \text { Estudo } \\
\text { prospectivo "antes } \\
\text { e depois" }\end{array}$ & $\begin{array}{l}\text { Avaliar se intervenções } \\
\text { em sono, sedação, } \\
\text { mobilização e dor têm } \\
\text { impacto na redução de da } \\
\text { duração de delirium em } \\
\text { pacientes internados em } \\
\text { UTI por lesão cerebral } \\
\text { aguda. }\end{array}$ & $\begin{array}{l}\text { Foram incluídos } 89 \text { pacientes com } 18 \\
\text { anos ou mais que tivessem lesão } \\
\text { cerebral aguda por hemorragia } \\
\text { traumática ou não traumática, com } \\
\text { previsão de permanência em UTI por } \\
48 \text { horas ou mais, que falassem } \\
\text { dinamarquês e que não tivessem lesão } \\
\text { cerebral prévia importante, } \\
\text { prognóstico letal ou persistência de } \\
\text { baixo grau de consciência. A média } \\
\text { de idade foi de } 62 \text { anos. Os pacientes } \\
\text { foram divididos entre os grupos } \\
\text { Standart Care e Intervention, sendo } \\
39 \text { para aquele e } 50 \text { para este. }\end{array}$ & $\begin{array}{l}\text { A prevalência de delirium foi de } 89 \% \text { na } \\
\text { população geral, não havendo diferença } \\
\text { estatística significativa entre os grupos. }\end{array}$ \\
\hline $\begin{array}{l}\text { FORD et al., 2020, } \\
\text { Ensaio Clínico } \\
\text { Randomizado } \\
\text { Duplo-cego } \\
\text { Placebo- } \\
\text { Controlado }\end{array}$ & $\begin{array}{l}\text { Verificar se a melatonina } \\
\text { é capaz de prevenir o } \\
\text { delirium pós-operatório } \\
\text { em pessoas submetidas a } \\
\text { cirurgias cardíacas de } \\
\text { alto porte. }\end{array}$ & $\begin{array}{l}210 \text { pessoas de } 50 \text { anos de idade ou } \\
\text { mais, com média de idade de } \\
\text { aproximadamente } 68 \text { anos, } \\
\text { submetidas a revascularização do } \\
\text { miocárdio e /ou substituição valvar. }\end{array}$ & $\begin{array}{l}\text { A pessoas foram divididas em um grupo- } \\
\text { intervenção, com a melatonina, e outro } \\
\text { placebo, sendo ambos compostos por } 105 \\
\text { indivíduos. A incidência de delirium foi } \\
\text { semelhante nos dois grupos. Por isso, os } \\
\text { resultados do estudo não corroboram com o } \\
\text { uso profilático da melatonina na prevenção de } \\
\text { delirium no pós-operatório de cirurgia } \\
\text { cardíaca. }\end{array}$ \\
\hline $\begin{array}{l}\text { BAUMGARTNER } \\
\text { et al., 2019, Estudo } \\
\text { de coorte } \\
\text { observacional } \\
\text { retrospectivo }\end{array}$ & $\begin{array}{l}\text { Verificar a performance } \\
\text { da } \\
\text { administrada melatonina } \\
\text { como à noite } \\
\text { farmacológico profilático } \\
\text { contra delirium em } \\
\text { pacientes internados em } \\
\text { UTI. }\end{array}$ & $\begin{array}{l}132 \text { pessoas, com média de idade } 60 \\
\text { anos. }\end{array}$ & $\begin{array}{l}\text { O grupo que recebeu a melatonina foi } \\
\text { composto por } 117 \text { pessoas, com incidência de } \\
\text { delirium de } 7,7 \% \text {; já o grupo controle, } \\
\text { composto por } 115 \text { pessoas, teve } 24,3 \% \text { de sua } \\
\text { população com delirium. Dessa forma, esse } \\
\text { estudo fortalece a hipótese do uso da } \\
\text { melatonina como agente profilático do } \\
\text { delirium em pacientes na UTI. Contudo, o } \\
\text { estudo destaca a necessidade de pesquisas } \\
\text { randomizadas que verifiquem essas } \\
\text { constatações. }\end{array}$ \\
\hline
\end{tabular}

Fonte: Autores.

Pacientes idosos internados em UTI são uma população com significativa prevalência de delirium, de forma que há diversas ferramentas clínicas de predição de risco e avaliação de gravidade desse evento de potencial morbidez. O Confusion Assessment Method (CAM) é um conjunto de critérios clínicos que podem ser aplicados e avaliados de forma multiprofissional para diagnosticar o delirium, auxiliando no seu manejo precoce, sendo reconhecido como um excelente método para este fim. Em sua forma adaptada à UTI, o CAM-ICU, em inglês, tem especificidade e sensibilidade importantes para a prática clínica. Essa ferramenta têm importância em detectar o risco de delirium e permitir que se aja em modificação aos fatores de risco, bem como de predizer a prevalência de delirium em determinados serviços em saúde, estimulando sua prevenção primária e possibilitando a evitar frequentes complicações (Coburn et al., 2018)(Page et al., 2017)(O’Connell et al., 2018)(de la Varga-Martínez et al., 2021).

Ainda, destaca-se que tem se estudado e publicado sobre a possibilidade de prevenção primária de delírio em idosos feita por fármacos. Nesse contexto, uma randomização com mais de 140 pacientes de ambos os sexos e com cerca de 62 anos 
de idade encontrou que a sinvastatina não diminuiu a incidência e a duração dos episódios de delirium na sua população, desencorajando a utilização clínica dessa medicação para este fim (Page et al., 2017).

Os estudos selecionados nessa revisão tiveram objetivos heterogêneos, de forma a diversificar o focos das suas conclusões; por exemplo, um deles (Coburn et al., 2018) avaliou, de forma multicêntrica e randomizada, qual agente anestésico - o xenônio ou o sevoflurano - seria melhor profilático de delirium para idosos com fratura de quadril, com média de idade de 84 anos e com posterior internação em UTI. Esse estudo concluiu que não houve diferença quanto à incidência de delirium pós-operatório no uso das duas medicações e indicou os benefícios de novas pesquisas sobre as hipóteses que ele elencou. (Coburn et al., 2018). Nessa mesma perspectiva ortopédica, uma coorte (O'Connell et al., 2018) de 144 idosos internados em UTI após cirurgia de reparo de trauma grave de tronco, com média de idade de 74 anos e meio apresentou que o delirium esteve prevalente em $32 \%$ da sua amostra e que as pessoas que receberam analgesia regional tiveram menor incidência de delirium do que as tratadas com opioides.

A cirurgia cardíaca também é uma área que tem se empenhado em buscar por prevenção primária de delirium em idosos internados em UTI. A exemplo disso, um estudo prospectivo (de la Varga-Martínez et al., 2021) com o intuito de validar um formulário capaz de predizer o risco pós-operatório de delirium e sua detecção precoce em pacientes submetidos a cirurgia cardíaca assumiu cerca de 690 indivíduos em dois grupos randomizados, um para formular os parâmetros e outro para validá-los. A média de idade dos pacientes incluídos nessa pesquisa foi de 70.7 anos, e concluiu-se que o DELIrium PREvention CArdiac Surgery (DELIPRECAS) pode predizer o risco do paciente elencado para cirurgia cardíaca desenvolver delirium pós-operatório; no Risco Moderado, a ferramenta apresentou sensibilidade 65,8 de e especificidade de 79,8. Foram usados 04 fatores de risco como parâmetro: Miniexame do Estado Mental indicativo de função cognitiva prejudicada ou pelo menos possibilidade de função cognitiva prejudicada; baixa frequência de atividade física; idade maior que 65 anos e presença de insônia que demanda tratamento. Além disso, associou o risco de desenvolvimento de delirium a fatores como maior tempo de intubação e maior tempo de internamento. Ainda, os autores ressaltaram que a prevenção primária do delirium é a melhor forma de tratá-lo.

Uma randomização multicêntrica (Hatta et al., 2017) verificou, em uma amostra de 72 pacientes de 65 a 89 anos, se o sulvorexant - um antagonista do receptor da orexina, comumente utilizado para o tratamento da insônia - seria superior ao placebo na prevenção do delirium ao atuar no equilíbrio do ciclo sono-vigília dos pacientes. A partir dessa hipótese, o estudo apresentou que o sulvorexant teve efeito protetivo contra o delirium, diminuindo sua incidência na população estudada, pois o grupo que o recebeu não teve casos de delirium, enquanto o placebo teve $17 \%$ da sua população acometida. Outra randomização colocou a Ramelteona - fármaco que também é geralmente utilizado para o tratamento de insônia - em prova como profilaxia oral ou via tubo de alimentação de delirium de pessoas com 20 anos ou mais internadas em UTI. Sua média de idade foi de 68 anos e encontrou-se que o grupo que não recebeu a ramelteona teve o dobro de incidência de delirium e maior tempo de internação, em comparação com o que recebeu. Outro estudo randomizado (Deiner et al., 2017), testou se o uso de dexmedetomidina intraoperatória teria impacto sobre a incidência de delirium pós-operatório, diminuindo-a. Encontrou-se que a incidência no grupo intervenção (12,2\%) foi semelhante ao do placebo (11,4\%). Um ensaio clínico (Van Den Boogaard et al., 2018) objetivou verificar qual o efeito do haloperidol como agente profilático de delirium em pacientes em UTI com alto risco predito de delirium.

A amostragem desse estudo consistiu em 1.789 pacientes com média de idade de 66,6 anos, e apresentou que o haloperidol teve desempenho semelhante ao placebo para esse objetivo. Também, uma coorte retrospectiva (Cucci et al., 2021) com 300 pessoas de média de idade 65,3 anos, objetivou avaliar se o período de tempo após admissão em UTI em que se reiniciava o uso de medicações neuropsiquiátricas teria influência sobre a incidência de delirium. Encontrou-se que a administração desses fármacos antes ou após três dias de internação não apresentaram diferença quanto ao aparecimento de 
novos casos de delirium. A melatonina foi estudada por dois de nossos artigos resgatados; um deles, um ensaio clínico randomizado (Ford et al., 2020), teve uma amostra de 210 pessoas, com média de idade de 68 anos, submetidas a cirurgias cardíacas e posteriormente internadas em UTI. Foi realizada profilaxia com melatonina em metade da população da pesquisa e não houve diferença de incidência de delirium com relação ao grupo que não recebeu essa droga, o que desencoraja o uso de melatonina para profilaxia de delirium em pessoas idosas internadas em UTI. Já outro estudo observacional retrospectivo (Baumgartner et al., 2019) com 132 pessoas internas em UTI com média de idade de 60 anos apresentou que a incidência de delirium no grupo que havia recebido a melatonina como profilaxia ao delirium foi de $7,7 \%$, enquanto as pessoas que não a haviam recebido estavam com prevalência de $24,3 \%$ de delirium. Contudo, este estudo ressaltou que randomizações seriam necessárias para melhor verificar a evidência desses achados.

Para além da profilaxia farmacológica do delirium, a prevenção primária pode ser feita de forma não-medicamentosa e os nossos resultados corroboram com isso. Uma análise de série temporal (van de Pol et al., 2017) com 411 participantes com média de idade de 68,5 anos apresentou que a redução do som durante à noite foi eficaz na diminuição da incidência do delirium na população estudada. Algumas das medias foram estas: não rir ou falar alto no saguão ou perto dos pacientes, baixar o volume dos alarmes sem comprometer a segurança do cuidado, deixar as portas fechadas quando o paciente não estivesse em delírio e fornecer tampões de ouvido aos pacientes à noite. Já uma pesquisa prospectiva (Rosa et al., 2017) teve o objetivo de observar quais os desdobramentos sobre a incidência do delirium seriam exercidos a partir do aumento do período de visitação de pacientes internos em UTI. Para isso, utilizou-se de um n de 286 pacientes, com média de idade de cerca de 61 anos e meio, dividido em dois grupos, um com visitação restrita $(n=141)$ e outro com visitação estendida $(n=145)$. O com restrições tinha a visita mais curta e com menor período disponível para acontecer (4 horas e meia), enquanto o outro grupo dispunha de mais tempo (12h), com visitas mais longas. Os resultados desse estudo denotaram o dobro de prevalência de delirium no grupo com restrições $(20,5 \%)$ em comparação com o outro (10\%), fortalecendo o uso dessa prática no cotidiano clínico. Ainda, medidas não-farmacológicas somadas a farmacológicas de prevenção do delirium ligadas ao sono, à dor, à sedação e à mobilização em pacientes idosos internos em UTI foi observada em um estudo prospectivo (Larsen et al., 2020) que não apresentou diferença estatística entre o grupo intervenção e o controle quanto à incidência desse adoecimento. Nessa pesquisa, usou-se a analgesia antes da sedação, visando a manter o paciente sem dor; para o sono, trocou-se a rotina de procedimentos que envolvem poluição sonora para o dia; utilizou-se uma escala de dor específica para pacientes incapazes de descrever a dor, a fim de tratála mesmo quando não autorrelatada; quanto à mobilização, o objetivo seria manter o paciente ativo o quanto fosse possível e saudável, segundo Balas e colaboradores (2012).

\section{Conclusão}

Diante do exposto nos resultados e discussão, conclui-se que há formas farmacológicas e não-farmacológicas que podem diminuir a incidência do delirium em pacientes idosos internados em UTI. Os estudos que embasam isso e foram resgatados nessa revisão têm diferentes escopos no que tange ao motivo do internamento dessa população, de forma que a eficácia evidenciada por essas pesquisas de determinadas medicações está diretamente relacionada ao fator causal do adoecimento dos determinados idosos. A respeito disso, tem-se que a melatonina não teve boa performance profilática contra o delírio na população submetida à cirurgia cardíaca, o que foi invertido em uma coorte que ampliou seus critérios e incluiu doentes severos - e inclusive procedentes de cirurgia cardíaca. Ainda, estudos observacionais foram resgatados para os resultados desse artigo e destacam em suas conclusões a necessidade de novas randomizações que elevem - ou não - o nível de evidência de seus achados finais. Com isso, reconhecendo as limitações desta revisão, destacamos que novas metanálises devem ser feitas sobre o escopo deste artigo para que se reúna evidências acerca do tema e a prevenção do delírium em idosos internados em UTI seja feita de forma mais eficaz, reduzindo complicações em saúde evitáveis para esta população. 


\section{Referências}

Balas, M. C., Vasilevskis, E. E., Burke, W. J., Boehm, L., Pun, B. T., Olsen, K. M., Peitz, G. J., \& Ely, W. (2012). Critical care nurses' role in implementing the "ABCDE Bundle" into practice. Critical Care Nurse, 32(2), 35-47. https://doi.org/10.4037/ccn2012229

Baumgartner, L., Lam, K., Lai, J., Barnett, M., Thompson, A., Gross, K., \& Morris, A. (2019). Effectiveness of Melatonin for the Prevention of Intensive Care Unit Delirium. Pharmacotherapy, 39(3), 280-287. https://doi.org/10.1002/phar.2222

Coburn, M., Sanders, R. D., Maze, M., Nguyên-Pascal, M. L., Rex, S., Garrigues, B., Carbonell, J. A., Garcia-Perez, M. L., Stevanovic, A., Kienbaum, P., Neukirchen, M., Schaefer, M. S., Borghi, B., van Oven, H., Tognù, A., Al Tmimi, L., Eyrolle, L., Langeron, O., Capdevila, X., ... Kunitz, O. (2018). The hip fracture surgery in elderly patients (HIPELD) study to evaluate xenon anaesthesia for the prevention of postoperative delirium: a multicentre, randomized clinical trial. British Journal of Anaesthesia, 120(1), 127-137. https://doi.org/10.1016/j.bja.2017.11.015

Cucci, M. D., Cunningham, B. S., Patel, J. S., Shimer, A. T., Mofleh, D. I., \& Mullen, C. L. (2021). Impact of Early Reinitiation of Neuropsychiatric Medications on Agitation and Delirium in the Intensive Care Unit: A Retrospective Study. Annals of Pharmacotherapy, 55(1), 15-24. https://doi.org/10.1177/1060028020935589

de la Varga-Martínez, O., Gómez-Pesquera, E., Muñoz-Moreno, M. F., Marcos-Vidal, J. M., López-Gómez, A., Rodenas-Gómez, F., Ramasco, F., ÁlvarezRefojo, F., Tamayo, E., \& Gómez-Sánchez, E. (2021). Development and validation of a delirium risk prediction preoperative model for cardiac surgery patients (DELIPRECAS): An observational multicentre study. Journal of Clinical Anesthesia, 69(November 2020). https://doi.org/10.1016/j.jclinane.2020.110158

Deiner, S., Luo, X., Lin, H. M., Sessler, D. I., Saager, L., Sieber, F. E., Lee, H. B., \& Sano, M. (2017). Intraoperative infusion of dexmedetomidine for prevention of postoperative delirium and cognitive dysfunction in elderly patients undergoing major elective noncardiac surgery : A randomized clinical trial. JAMA Surgery, 152(8), e171505. https://doi.org/10.1001/jamasurg.2017.1505

Estrela, C. (2018). Metodologia Científica: Ciência, Ensino, Pesquisa. Editora Artes Médicas.

Faustino, T. N., Pedreira, L. C., Freitas, Y. S. de, Silva, R. M. de O., \& Amaral, J. B. do. (2016). Prevenção e monitorização do delirium no idoso: uma intervenção educativa. Revista Brasileira de Enfermagem, 69(4), 725-732. https://doi.org/10.1590/0034-7167.2016690416i

Ford, A. H., Flicker, L., Kelly, R., Patel, H., Passage, J., Wibrow, B., Anstey, M., Edwards, M., \& Almeida, O. P. (2020). The Healthy Heart-Mind Trial: Randomized Controlled Trial of Melatonin for Prevention of Delirium. Journal of the American Geriatrics Society, 68(1), 112-119. https://doi.org/10.1111/jgs.16162

Hatta, K., Kishi, Y., Wada, K., Takeuchi, T., Ito, S., Kurata, A., Murakami, K., Sugita, M., Usui, C., \& Nakamura, H. (2017). Preventive effects of suvorexant on delirium: A randomized placebo-controlled trial. Journal of Clinical Psychiatry, 78(8), e970-e979. https://doi.org/10.4088/JCP.16m11194

Herling, S., Greve, I., Vasilevskis, E., Egerod, I., Bekker Mortensen, C., Møller, A., Svenningsen, H., \& T, T. (2018). Interventions for preventing intensive care unit delirium in adults ( Review ). Cochrane Library [revista en Internet] 2018 [acceso 25 de noviembre de 2019]; 11:1-94. https://doi.org/10.1002/14651858.CD009783.pub2.www.cochranelibrary.com

Koche, J. C. (2011). Fundamentos de metodologia científica. Petrópolis: Vozes. Disponível em: http://www.brunovivas.com/wpcontent/uploads/sites/10/2018/07/K\%C3\%B6che-Jos\%C3\%A9-Carlos0D0AFundamentos-de-metodologia-cient\%C3\%ADfica-_-teoriada0D0Aci $\%$ C3\%AAncia-e-inicia\%C3\%A7\%C3\%A3o-\%C3\%A0pesquisa.pdfhttps://repositorio.ufsm.br/bitstream/handle/1/15824/Lic_Computacao_Metodologia-Pesquisa-Cientifica.pdf?sequence=1

Larsen, L. K., Møller, K., Petersen, M., \& Egerod, I. (2020). Delirium prevalence and prevention in patients with acute brain injury: A prospective before-andafter intervention study. Intensive and Critical Care Nursing, 59(xxxx), 102816. https://doi.org/10.1016/j.iccn.2020.102816

Ludke, M. \& Andre, M. E . D. A. (2013). Pesquisas em educação: uma abordagem qualitativa. São Paulo: E.P.U.

O’Connell, K. M., Quistberg, D. A., Tessler, R., Robinson, B. R. H., Cuschieri, J., Maier, R. V., Rivara, F. P., Vavilala, M. S., Bhalla, P. I., \& Arbabi, S. (2018). Decreased risk of delirium with use of regional analgesia in geriatric trauma patients with multiple rib fractures. Annals of Surgery, 268(3), 534-540. https://doi.org/10.1097/SLA.0000000000002929

Page, V. J., Casarin, A., Ely, E. W., Zhao, X. B., McDowell, C., Murphy, L., \& McAuley, D. F. (2017). Evaluation of early administration of simvastatin in the prevention and treatment of delirium in critically ill patients undergoing mechanical ventilation (MoDUS): a randomised, double-blind, placebo-controlled trial. The Lancet Respiratory Medicine, 5(9), 727-737. https://doi.org/10.1016/S2213-2600(17)30234-5

Pereira A. S. et al. (2018). Metodologia da pesquisa científica. [free e-book]. Santa Maria/RS. Ed. UAB/NTE/UFSM.

Rosa, R. G., Tonietto, T. F., Da Silva, D. B., Gutierres, F. A., Ascoli, A. M., Madeira, L. C., Rutzen, W., Falavigna, M., Robinson, C. C., Salluh, J. I., Cavalcanti, A. B., Azevedo, L. C., Cremonese, R. V., Haack, T. R., Eugênio, C. S., Dornelles, A., Bessel, M., Teles, J. M. M., Skrobik, Y., \& Teixeira, C. (2017). Effectiveness and safety of an extended icu visitation model for delirium prevention: A before and after study. Critical Care Medicine, 45(10), 16601667. https://doi.org/10.1097/CCM.0000000000002588

Ursi, E. S. (2005). Prevenção de lesões de pele no perioperatório: revisão integrativa da literatura. Revista Latino-Americana de Enfermagem, $14(1)$, 2-127. van de Pol, I., van Iterson, M., \& Maaskant, J. (2017). Effect of nocturnal sound reduction on the incidence of delirium in intensive care unit patients: An interrupted time series analysis. Intensive and Critical Care Nursing, 41, 18-25. https://doi.org/10.1016/j.iccn.2017.01.008

Van Den Boogaard, M., Slooter, A. J. C., Brüggemann, R. J. M., Schoonhoven, L., Beishuizen, A., Vermeijden, J. W., Pretorius, D., De Koning, J., Simons, K. S., Dennesen, P. J. W., Van Der Voort, P. H. J., Houterman, S., Van Der Hoeven, J. G., \& Pickkers, P. (2018). Effect of haloperidol on survival among critically ill adults with a high risk of delirium the REDUCE randomized clinical trial. JAMA - Journal of the American Medical Association, 319(7), 680 690. https://doi.org/10.1001/jama.2018.0160

Yin, R.K. (2015). O estudo de caso. Porto Alegre: Bookman. 